\title{
Pattern goodness and speed of encoding
}

\author{
JAMES R. POMERANTZ \\ Yale University, New Haven, Connecticut 06520
}

\begin{abstract}
Two experiments attempted to resolve a conflict in the literature about whether good patterns are encoded faster than poor patterns in speeded classification tasks. The results showed clear effects of goodness on the memory component of such tasks, but no effect on speed of encoding. The conflicts appear to be due in part to varying encoding requirements of different tasks.
\end{abstract}

Pattern goodness, as described by the Gestalt psychologists, is a subjectively compelling property of visual configurations. Goodness also has clearly observable effects in the performance of simple perceptual and memory tasks. For example, good patterns are easier to discriminate, describe, and remember than are poor patterns (Attneave, 1955; Clement, 1964; Clement \& Varnadoe, 1967).

The present study is aimed at pinpointing the locus of the effects of goodness in information processing. If it is assumed that performance in any task can be reduced to a series of temporally ordered stages, the problem is identifying the stage (or stages) which is affected by pattern goodness.

For stimulus classification tasks, two broad stages of processing may be distinguished, one concerned with the encoding of incoming stimuli, and the other concerned with storage in and retrieval from memory. Encoding is involved with transforming inputs into a form suitable for comparison with memory items, and so is affected by factors such as stimulus intensity and clarity. The memory stage is concerned with retrieving relevant items from memory and comparing them to the encoded stimulus. Thus, this stage is affected by factors such as the number and similarity of the alternative stimuli to be classified (Sternberg, 1967, 1969).

Clement and Varnadoe (1967) examined the role of pattern goodness in a simple two-choice discrimination task. Subjects were presented with dot patterns of varying goodness, one at a time, and were asked to make a speeded choice response. In any one condition, only two patterns could appear, with each assigned to a different response. The two patterns could both be good

This paper is based on a portion of a doctoral dissertation submitted to Yale University. The author thanks W. R. Garner, his dissertation advisor, and R. Abelson, R. Crowder, R. Day, $M$. Kubovy, and M. Posner, all members of his dissertation committee, for their help. Thanks also go to Irving Biederman and to Howard Egeth for their comments on an earlier draft of this paper. This research was supported by Grant MH 14229 from the National Institute of Mental Health to Yale University. Send reprint requests to the author at the Department of Psychology, Johns Hopkins University, Baltimore, Maryland 21218. ones (denoted as $\mathrm{G} / \mathrm{G}$ ), both be poor $(\mathrm{P} / \mathrm{P})$, or be one good and one poor $(\mathrm{G} / \mathrm{P})$. The results showed the $\mathrm{G} / \mathrm{G}$ discrimination to be the fastest, $\mathrm{P} / \mathrm{P}$ the slowest, and $\mathrm{G} / \mathrm{P}$ midway between.

It is not possible, on the basis of this study alone, to localize the effect of goodness to encoding or to memory processes, since both possibilities are plausible. For example, if good patterns were encoded faster than poor ones, this alone could explain the obtained ordering of the three conditions; $G / G$ was fastest because both stimuli could be encoded quickly, while G/P was somewhat slower because only one of the patterns could be encoded quickly, and so on. On the other hand, the results could be ascribed solely to the memory component of the task. In order to perform properly, the subject had to remember which stimuli were possible (along with the correct response assignments). If good patterns are easier to hold in or retrieve from memory, then the memory component of the $\mathrm{G} / \mathrm{G}$ task would be easiest, G/P intermediate, and $\mathrm{P} / \mathrm{P}$ hardest.

Garner and Sutliff (1974) suggested that the two possibilities might be differentiated by examining reaction times (RTs) to individual patterns, which Clement and Varnadoe did not do. If good patterns are encoded faster than poor ones, then RTs should be faster to the good than to the poor pattern in the G/P task. Garner and Sutliff's results confirmed this prediction, and they interpreted this to indicate an effect of goodness on speed of encoding (which they defined broadly to include simple registration, placement in short-term store, or even response readiness).

Further support for this encoding-time hypothesis was reported by Bell and Handel (1976). They found that good patterns were reproduced more accurately than were poor patterns under backward masking conditions, although no difference appeared without masking. From these and other results, they concluded that the good patterns were encoded faster and so were more likely to be encoded fully before the onset of the mask.

\section{Checkosky and Whitlock Study}

On the other hand, an experiment by Checkosky and Whitlock (1973) raises some doubts about the encoding- 
time hypothesis. Theirs was a memory scanning task, again using good and poor dot patterns as stimuli. The goodness of the patterns in the memory (positive) set was varied independently of the goodness of probe stimuli. In addition, probes were presented under conditions of high or low contrast (visibility).

Checkosky and Whitlock analyzed for encoding and memory effects by using Sternberg's (1969) additive factors logic, which holds that factors affecting different stages of processing should show additive effects on RT. They found that probe goodness and probe contrast did not interact. Since probe contrast should affect encoding time, they concluded that probe goodness did not affect the encoding stage. However, memory-set goodness did interact with memory-set size, such that poor patterns yielded larger set-size effects. This was taken as evidence of a memory locus of goodness.

Checkosky and Whitlock's conclusion that goodness does not affect encoding assumes that the encoding process is a single, unitary stage. It is possible, however, to construct two-stage models in which probe contrast and probe goodness affect encoding time in an additive manner. Consider, for example, a model in which sensory receptors signal the presence of a stimulus by sending impulses at a fixed speed along pathways to a set of memory representations or logogens (Morton, 1969). Each logogen, which corresponds to a particular stimulus pattern, accumulates information about the stimulus and fires whenever its preset criterion is exceeded. The firing of a logogen corresponds to the encoding of the stimulus. Suppose probe contrast affects the rate at which impulses are generated at the receptor level (Stage 1) and that probe goodness determines the length of the pathway from the receptors to the logogen (Stage 2) with poor patterns requiring, say, more feature tests before making contact with memory. This hypothetical model predicts that both goodness and contrast will affect RT, but in an additive way, since the two factors affect separate, sequential substages of encoding. Thus, this model explains the data of Checkosky and Whitlock by maintaining that probe goodness affects only the second stage of encoding, while probe contrast affects only the first stage. ${ }^{1}$

Although this model is speculative and is less parsimonious than a model positing only a single stage of encoding, it can explain another aspect of Checkosky and Whitlock's data. Stemberg (1967) argued that factors affecting the encoding stage should influence the intercept of the function relating RT to the size of the positive set. Garner (1974) reanalyzed Checkosky and Whitlock's data in this manner and found, as the model predicts, that goodness did affect the intercept, with poor probes showing higher intercepts than did good probes. While this result is open to several interpretations (since other stages besides encoding can influence the intercept), it is certainly consistent with the notion that goodness affects speed of encoding.

\section{Logic of Present Study}

To summarize, all of the data from the four experiments reviewed above are equivocal with respect to the hypothesis that pattern goodness affects encoding time. That is, all the data are consistent with the hypothesis, but none of the data compel it. While an additive factors experiment might seem to be the best way to resolve the matter, this approach rests on the assumptions that two factors affecting the encoding stage must interact (which, as Sternberg has argued, is false), and that encoding is a unitary stage as opposed to a collection of substages (an assumption that the additive factors method cannot test directly). The experiments presented below were aimed at testing the encoding-time hypothesis in a more direct manner. More specifically, they sought to determine whether there are any effects of probe goodness on RT when the goodness of the patterns in memory is held constant; if not, it can be safely concluded that goodness does not affect encoding time. Thus, the principal manipulations of these experiments were designed to control the contents of the subject's memory set.

Even in the simple two-choice discrimination task, it is not usually clear which items the subject holds in memory. Consider the G/P task. When the good pattern appears, one key should be pressed, while a second key should be pressed for the poor pattern. A subject might hold both patterns in memory, but he could also manage simply by remembering only one of them (i.e., by forming a positive set consisting of only one item). Probes could then be classified on the basis of their matching or mismatching the single "target" pattern. Thus, three different positive sets are logically possible for the G/P task: one good pattern, one poor pattern, or both patterns. Without knowing exactly which set was used, it is impossible to determine the effect of goodness on the memory component of the task. In addition, individual RTs to the good and poor pattern become impossible to interpret. For example, Garner and Sutliff's (1974) finding that the good pattern shows the faster RTs in the G/P discrimination does not necessarily imply faster encoding for good patterns. Suppose their subjects held only the good pattern in memory. Faster RTs to the good pattern would then correspond to the common finding that positive responses are faster than negative ones. This phenomenon could be ascribed to response biases, or to different processing mechanisms underlying positive and negative responses (Bamber, 1969), rather than to faster encoding of good patterns.

The logic of the present experiments was to examine RTs to good and poor patterns in tasks in which it is reasonably clear which patterns are held in memory. Experiment 1 tested whether memory strategies could be controlled in the two-stimulus task simply by instructing subjects to use just one of the patterns as the positive set. If so, this would permit encoding-time 
effects to be disentangled from memory effects and response biases.

\section{EXPERIMENT 1}

\section{Method}

Stimuli. The 10 stimuli used are shown in Figure 1. The goodness of the patterns may be defined either in terms of symmetry or in terms of the number of different patterns generated when the stimuli are reflected or rotated in increments of $90 \mathrm{deg}$ (see Garner \& Clement, 1963). Patterns were displayed with a 5 by 5 array of miniature light bulbs, which were covered with a dark translucent panel that kept the bulbs from being visible except when lit. Particular patterns were generated by illuminating subsets of nine bulbs. At a viewing distance of $150 \mathrm{~cm}$, the patterns subtended about $3 \mathrm{deg}$ of visual angle.

Conditions. From the 10 stimuli used, 45 different pairs can be drawn. The resulting 45 different pairwise discrimination tasks comprised this experiment. These 45 can be grouped into three different types of conditions: discrimination between two good patterns $(G / G)$, between two poor patterns $(P / P)$, and between one good and one poor (G/P). Of the 45 tasks employed, 10 were $G / G, 10$ were $P / P$, and 25 were G/P. In each of the tasks, only two patterns could appear on the display panel, one calling for a left keypress and one for a right. Each pattern occurred equally often in each of the 45 presentation sequences, which were 72 exposures long.

Groups. Two groups of five subjects each were used, and they differed only in the instructions the subjects received. Before each discrimination task, subjects in the neutral group were given exemplars for each of the two possible stimuli, which they placed next to the proper response keys, according to instructions. They were simply instructed to press the appropriate button as quickly and accurately as possible after a pattern appeared on the display. Subjects in the biased group were given an exemplar of only one of the two possible patterns, which they placed next to the proper response key. Although these subjects knew only two patterns were possible, they were asked to "focus their attention" on the exemplar pattern, to press the appropriate key whenever it appeared, and to press the other key whenever "any other" pattern appeared.

Design and procedure. Each subject was tested once on each of the 45 tasks, but in a different order, counterbalanced over subjects. The three types of conditions were mixed through this order. The assignment of patterns to response keys was also counterbalanced across subjects. Each task began when the subject signaled he was ready. Probe patterns then appeared on the display one at a time, remaining on until a response was made. The display then went blank for $1 \mathrm{sec}$ before the next pattern appeared. The two patterns appeared in a pseudorandom order, subject to the constraints that each pattern occur exactly 36 times, and that no pattern repeat itself more than four times

GOOD PATTERNS:
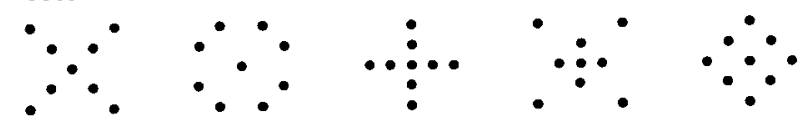

POOR PATTERNS:
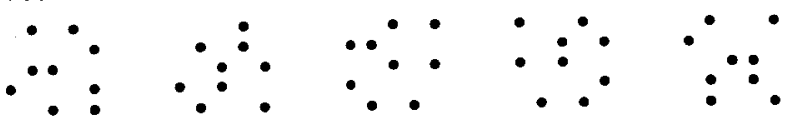

Figure 1. The 10 patterns used in the present experiments.
Table 1

RTs and Error Rates for Experiment 1

\begin{tabular}{lrcr} 
& \multicolumn{3}{c}{ a. Discrimination } \\
& G/G & G/P & P/P \\
\hline RT (Milliseconds) & 382 & 397 & 410 \\
Percent Errors & 2.4 & 2.6 & 3.0 \\
\hline & \multicolumn{3}{c}{ b-c. Probe Pattern } \\
& Good & Poor & Mean \\
\hline RT (Milliseconds) & 393 & 401 & \\
Percent Errors & 2.3 & 2.8 & \\
Bias on Good & 390 & 403 & 396 \\
Bias on Poor & 401 & 401 & 401 \\
Mean & 396 & 402 & \\
\hline
\end{tabular}

Note-(a) Data shown are collapsed across both groups of subjects for the three types of discrimination task. (b) Data are shown separately for the good and poor pattern in the G/P task only. (c) RT data shown are for the biased group only in the $G / P$ tasks.

in a row. Stimulus presentation and response recording were automated.

Subjects. Ten paid voluntecrs (one female) were used. The average age was 25 years and all subjects were college graduates. Each was tested individually.

\section{Results}

Table 1a shows mean correct RTs and error rates for the three conditions, collapsed over the two groups. The data are based on the last 60 stimuli in the exposure sequence of 72. The order of RTs for the three conditions shows $G / G$ faster than $G / P$, which was faster than $\mathrm{P} / \mathrm{P}[\mathrm{F}(2,18)=14.3, \mathrm{p}<.001]$. All three means differed significantly from each other $(p<.05$, Newman-Keuls test). Error rates increased in a parallel fashion.

Table $1 \mathrm{~b}$ shows separate RTs and errors for the good and poor pattern in the G/P condition. The good pattern was responded to $8 \mathrm{msec}$ faster than the poor one. Despite the small size of this effect, it reached significance $[t(9)=2.22, p<.05]$. Note, however, that the small RT effect was accompanied by a small difference in error rate; fewer errors were made when the good pattern appeared. While the error difference was based on too few data to reach significance, the general pattern of RTs and errors was consistent with a simple responsebias notion, whereby subjects were predisposed toward the good pattern.

Comparative data for the neutral and biased groups are not shown because they did not differ significantly in any respect, with regard to either RTs or errors. For the biased group considered alone, the pattern toward which they were "biased" was responded to $8 \mathrm{msec}$ faster than the unbiased pattern, but this difference was not significant $[F(1,4)=2.01, p>.10]$. Table $1 \mathrm{c}$ shows RT data for the biased group in the G/P tasks alone. If effective, the instructions would have had their greatest impact on these tasks: Overall RTs should 
have been faster when subjects focused their attention on (i.e., held in memory) the good pattern, rather than the poor one. While this was true (396 vs $401 \mathrm{msec}$ ), the difference did not approach significance. Table 1c also shows the same weak $(8 \mathrm{msec})$ encoding-time effect associated with probe goodness mentioned above.

\section{DISCUSSION}

This experiment successfully replicated the original goodness effect of Clement and Varnadoe (1967) and also replicated the finding of Garner and Sutliff (1974) that individual RTs are faster to good patterns than to poor patterns. The data, however, are equivocal with respect to the encoding-time hypothesis. The overall ordering of speed, $G / G>G / P>P / P$, can be explained by memory effects of goodness alone, as argued earlier. Faster responses to the good pattern in the G/P condition might have been due to faster encoding of the good pattern, reducing RT by a constant, but, in view of the lower error rate to the good pattern, this effect may simply involve a response bias. The attempt to dissociate encoding effects from response biases with the focusing instructions did not succeed; subjects were apparently reluctant to follow instructions that told them to use a strategy they perceived to be inefficient.

\section{EXPERIMENT 2}

Experiment 2 sought to manipulate memory strategies by varying the number of stimuli assigned to the two responses. If a single pattern were assigned to one response while several were assigned to the other, it would be expected that the subject would treat the single pattern as a target, in other words, use it as the positive set. If this expectation is correct, then RT should be independent of the number of patterns in the negative set (Sternberg, 1966). This experiment, therefore, had three major factors: (1) the goodness of the target stimulus (i.e., the one assigned to its own response); (2) the goodness of the nontarget stimuli (assigned to the second response); and (3) the number of nontarget stimuli (i.e., the size of the negative set), which was either two or four. These three binary factors resulted in eight different discrimination tasks.

The effect of goodness on the memory component of RT should be determined by the goodness of the target stimulus held in memory, holding constant the goodness of the probes. Conversely, the effect of goodness on encoding should be determined by the goodness of probe stimuli holding constant the goodness of the target in memory.

\section{Method}

Stimuli and conditions. The stimuli and the method of presentation were the same as in Experiment 1. The eight discrimination tasks used, which were outlined above, are shown in Table 2. The notational system for these tasks follows that used earlier. For example, G/PP indicates that three patterns were possible; the good pattern was the target and called for one response, while two different poor patterns were nontargets and either called for the second response.

Design and procedure. In most respects, the procedure was the same as in Experiment 1. Each subject participated once in each task, with the order counterbalanced over subjects. Before each task began, the subject was handed exemplars of all patterns that would appear in the discrimination. He placed one of these (the target) next to one response key and the others (nontargets) next to the other key. No instructions as to memory strategies were given. When the subject was ready, the stimulus sequence began. The sequence consisted of 144 probes. The target appeared on half of the exposures, and each of the two or four nontargets appeared equally often. The exemplars remained in view during the sequence. The sequences themselves were pseudorandom, subject to the constraints imposed by the probability of occurrence of the stimuli and by the constraint that no response should be repeated more than four times in a row.

In any one condition, each subject saw a different set of particular dot patterns, selected to balance the frequency with which any pattern appeared (1) in any discrimination, and (2) as a target stimulus. The assignment of patterns to the two responses was balanced over subjects, as was the assignment of the target to the two responses.

Subjects. A new group of 10 paid volunteers ( 2 female) was used. The average age was 20 years and all subjects were either enrolled in or graduated from college.

\section{Results}

Table 2 shows mean correct RTs and error rates for eight conditions. These data are based on the last 120 stimuli in each exposure sequence. The table shows data for both the target and nontarget probes in each discrimination, as well as overall data for the tasks as a whole, which will be discussed first. The data were submitted to an analysis of variance containing four

Table 2

RTs and Error Rates for Experiment 2

\begin{tabular}{|c|c|c|c|}
\hline \multirow[b]{2}{*}{ Condition } & \multicolumn{2}{|c|}{ Patterns } & \multirow{2}{*}{$\begin{array}{c}\text { Condition } \\
\text { Total } \\
\end{array}$} \\
\hline & Target & Nontarget & \\
\hline G/GG & $\begin{array}{l}426 \\
(3.8)\end{array}$ & $\begin{array}{c}449 \\
(3.8)\end{array}$ & $\begin{array}{c}438 \\
(3.8)\end{array}$ \\
\hline G/PP & $\begin{array}{c}397 \\
(4.7)\end{array}$ & $\begin{array}{c}425 \\
(2.5)\end{array}$ & $\begin{array}{c}411 \\
(3.6)\end{array}$ \\
\hline P/GG & $\begin{array}{c}457 \\
(5.5)\end{array}$ & $\begin{array}{c}453 \\
(3.2)\end{array}$ & $\begin{array}{c}455 \\
(4.3)\end{array}$ \\
\hline $\mathbf{P} / \mathbf{P P}$ & $\begin{array}{c}454 \\
(3.5)\end{array}$ & $\begin{array}{c}456 \\
(3.0)\end{array}$ & $\begin{array}{c}455 \\
(3.2)\end{array}$ \\
\hline G/GGGG & $\begin{array}{c}412 \\
(3.8)\end{array}$ & $\begin{array}{c}430 \\
(2.8)\end{array}$ & $\begin{array}{c}421 \\
(3.3)\end{array}$ \\
\hline G/PPPP & $\begin{array}{c}413 \\
(3.2)\end{array}$ & $\begin{array}{c}442 \\
(3.7)\end{array}$ & $\begin{array}{c}428 \\
(3.4)\end{array}$ \\
\hline P/GGGG & $\begin{array}{c}454 \\
(3.5)\end{array}$ & $\begin{array}{c}465 \\
(3.8)\end{array}$ & $\begin{array}{c}460 \\
(3.7)\end{array}$ \\
\hline P/PPPP & $\begin{array}{c}445 \\
(5.2)\end{array}$ & $\begin{array}{c}439 \\
(1.5)\end{array}$ & $\begin{array}{c}441 \\
(3.3)\end{array}$ \\
\hline
\end{tabular}

Note-RTs are in milliseconds for correct responses only; error rates, contained within parentheses below the corresponding $R T s$, are in percentages. 
binary factors: (1) goodness of the target stimulus, (2) goodness of the nontarget stimuli, (3) number of nontargets, and (4) the response itself, either to the target or to the nontargets.

Number of nontargets. Consider first the top vs bottom half of Table 2 . The analysis revealed no significant effect of the number of nontargets $[F(1,9)<1)]$. In fact, RTs were slightly faster for conditions with four nontargets than for those with two ( 438 vs $440 \mathrm{msec}$ ). The error data show the same effect. This indicates that the nontargets were treated as a genuine negative set and, hence, that the positive set consisted only of the target pattern. As discussed above, this allows RTs to be examined with memory load held constant.

Goodness of target. Compare next Rows 1,2,5, and 6 vs Rows $3,4,7$, and 8 . The analysis showed that the goodness of the target had a significant effect: When it was a good pattern, overall RTs were $29 \mathrm{msec}$ faster than when it was poor $[F(1,9)=9.35, p<.05]$. The error analysis shows a similar advantage for good targets. This result indicates that the task was generally easier when good patterns were in memory instead of poor ones, which parallels the finding of Checkosky and Whitlock (1973).

Goodness of nontargets. The effect of nontarget goodness may be seen by comparing the even- with the odd-numbered rows in Table 2 . The analysis revealed that this factor had no significant effect, although overall RTs were in fact $10 \mathrm{msec}$ faster when the nontargets were poor $[F(1,9)=1.34, p<.10]$. Again, the error data show a parallel result. Furthermore, there was no interaction of target and nontarget goodness $[F(1,9)<1]$. $^{2}$

The implications of the data are straightforward. First, pattern goodness clearly affected the memory component of these discrimination tasks. Second, the absence of any effect of nontarget goodness implies that goodness did not affect encoding time, at least for nontarget probes (see below for a discussion of target probes). If poor patterns had been encoded more slowly than good ones, then tasks for which the nontargets were poor should have been slower than tasks for which they were good, holding target goodness constant.

The preceding analyses have been based on overall RTs for each task, collapsed over responses to target and nontarget probes. Table 3a shows RTs to nontarget probes only, as a function of the goodness of the target pattern. As the table shows, RTs to good nontarget probes were no faster than to poor ones; in fact, they were somewhat slower and showed a higher error rate. This result corroborates the conclusion from the preceding analyses that goodness did not affect encoding time for nontargets.

Does goodness affect encoding time for targets? Demonstrating that goodness does not affect encoding speed for nontargets provides only a partial answer to the question at hand. One might argue that nontargets
Table 3

RTs to Good and Poor Probes as a Function of Target Goodness in Experiment 2

\begin{tabular}{|c|c|c|c|c|c|}
\hline & & \multicolumn{4}{|c|}{ Target (Memory) Pattern } \\
\hline & & \multicolumn{2}{|c|}{ Good } & Poor & Mean \\
\hline & & \multicolumn{4}{|c|}{ a. RTs to Nontarget Probes } \\
\hline Probe & Good & 440 & $(3.3)$ & $459 \quad(3.5)$ & 449 \\
\hline \multirow[t]{3}{*}{ Pattern } & Poor & 434 & $(3.1)$ & $448 \quad(2.2)$ & 441 \\
\hline & Mean & 437 & & 453 & \\
\hline & & \multicolumn{4}{|c|}{ b. RTs to Target Probes } \\
\hline Probe & Good & 412 & $(3.9)$ & & 412 \\
\hline \multirow{2}{*}{ Pattern } & Poor & & & $452 \quad(4.4)$ & 452 \\
\hline & Mean & 412 & & 452 & \\
\hline
\end{tabular}

Note-RTs are in milliseconds for correct responses only: error rates, contained within parentheses beside the corresponding $R T s$, are in percentages.

are not as fully encoded as are targets in a classification task. Thus, it is important to determine if goodness affects encoding time for target probes. The relesant analysis is given in Table $3 b$. Note that only two of the four cells of this table can be filled. This is because, when only responses to targets are considered, target goodness and probe goodness are necessarily confounded. The table shows significantly faster RTs to good targets than to poor ones. Because of the confounding in the table, this difference can be interpreted in either of two ways. First, it could be regarded as an effect of probe goodness (a row effect), which would imply that good targets were encoded faster than were poor ones. Alternatively, it could be regarded as an effect of target goodness (a column effect); that is, it could simply mean that the memory requirements of the task were greater when a poor pattern comprised the positive set. Supporting this memory interpretation is the fact that a similar column effect appeared with nontargets in Table 3a, where probe goodness and target goodness are unconfounded $[\mathrm{t}(9)=1.92, \mathrm{p}<.05]$. That is, RTs to nontarget probes were faster when the target pattern in memory was a good pattern. Thus, it appears that at least part of the effect with targets shown in Table $3 b$ is due to memory factors. Whether the small difference that remains (after memory factors are removed) is due to faster encoding time for good targets than for poor targets is uncertain; it could just as likely have been due to response-bias variations. This point is a complex one and requires some clarification. First, note that RTs to targets (Table $3 \mathrm{~b}$ ) were generally faster than to nontargets (Table 3a). This corresponds to the common finding in the literature that positive responses are faster than negative responses. Second, closer inspection shows that this was true only when the target was a good pattern (25-msec effect), not when it was a poor one (1-msec effect). This interaction proved to be significant $[F(1,9)=8.94, p<.05]$. Analysis of the corresponding error rates suggests that 
this difference may have been due to shifts in response bias. In tasks where the target was a poor pattern, more errors were made to target probes than to nontarget probes, which would be expected if subjects were biased toward the nontarget response in these conditions. The effect of this bias would be to lengthen RTs to poor target probes, which in turn would exaggerate the row effect in Table $3 \mathrm{~b}$. It is beyond the resolving power of this experiment to determine whether any significant row effect exists in Table $3 b$ after both the response-bias factor and the memory factor (column effect) discussed above have been removed. In summary, goodness may have no effect on encoding time for target probes, but if it does have an effect, it is certain to be a small one.

\section{Discussion}

To summarize, the results of Experiment 2 have shown clearly that nontarget patterns were encoded no faster when they were good than when they were poor. The same is probably true for target patterns, as well, although the data cannot answer this question definitively.

\section{CONCLUSIONS}

Failure to find effects of pattern goodness on the encoding of nontargets might be interpreted to mean that nontargets are not encoded to any significant degree in classification tasks. Neisser (1967), for example, has argued that in search tasks, subjects process nontargets only minimally, to a level where they know only that the stimulus is not the target, but do not know the actual identity of the stimulus. Target stimuli, however, are encoded more deeply, up to the level where they are positively identified. Therefore, it could be concluded that goodness facilitates only deeper levels of encoding reserved for target probes. Experiment 2 does not support this explanation, however. First, there was no strong evidence that the encoding of targets was affected by goodness. Second, this experiment does contain evidence that nontargets were encoded to a significant degree. Specifically, a separate analysis showed consistent repetition effects (Kornblum, 1973) for nontargets in all eight conditions of the experiment. That is, when a particular nontarget pattern occurred twice in a row in the stimulus sequence, RTs to the second occurrences were shorter than to the first (by $24 \mathrm{msec}$, on the average). This suggests that nontargets were being recognized at least on the second successive appearance. Third, if nontargets were only partially encoded in this experiment, one would expect them to show shorter RTs than the more fully encoded target probes. The data do not confirm this prediction, although, once again, response biases toward the target could have masked this effect. In any case, nontarget probes must logically be encoded to some degree before they can be recognized as nontargets. The data show clearly that the speed of the encoding process is unaffected by pattern goodness.

\section{Explaining the Results of Previous Experiments}

The main conclusion of this study, then, is that good patterns are encoded no faster than poor patterns in a speeded classification task. Thus, this conclusion is in agreement with that reached by Checkosky and Whitlock (1973), and it argues against the alternative, two-stage encoding model presented earlier to explain their results. How is this conclusion to be reconciled with previous data that implied an effect of goodness on encoding? First, Garner and Sutliff's (1974) finding of faster RTs to good than to poor patterns in the G/P task is probably due to a response bias in favor of the good pattern. Their subjects apparently used the good pattern as a target (i.e., as their positive set), and, thus, the result is consistent with the usual superiority of positive over negative responses. Second, the intercept effect of probe goodness that Garner discovered in Checkosky and Whitlock's data, if not a chance occurrence, might be ascribed to some process, other than encoding, that affects the intercept, such as decision or response selection.

\section{When Does Goodness Affect Encoding?}

Third, Bell and Handel's (1976) finding of an effect of goodness on encoding was probably caused by different encoding demands in their task from those in classification tasks. Encoding, as used in the present paper, refers to whatever operations must be applied to the stimulus input in order to perform the task. Bell and Handel's task was one of pattern reproduction, which required subjects to store test patterns in memory long enough to reproduce them a few moments later. Their finding that good patterns are more resistant to masking may mean that good patterns can be encoded more rapidly into a relatively long-lasting memory code, an operation not required in simple discrimination.

Apparently, goodness does not matter for the kind of encoding required in a speeded discrimination task. This result is sensible if the encoding process for the task is a primitive one, such as template formation, or a point-by-point transformation that is insensitive to the complexity or meaning of the stimulus being encoded. This template or "blob" (Lockhead, 1972) could then be matched immediately against a memory representation of the target pattern. On the other hand, there are many perceptual tasks in which deeper levels of encoding take place. For example, when a task requires encoding a stimulus into a form suitable for storage in and retrieval from memory, a more detailed analysis of stimulus structure may take place. Good patterns are redundant (Bear, 1973; Garner, 1970), and so can be specified with a smaller number of features than can poor patterns. This allows good 
patterns to be encoded into memory in a shorter time. For a second example, the perceptual organization of meaningful stimuli can also be strongly affected by goodness, as the Gestalt psychologists argue. Classical demonstrations such as the Ames Room or the trapezoidal window show clearly that meaningful patterns will be encoded in the best possible way. More recent experiments on performance in information processing tasks (Pomerantz \& Garner, 1973; Pomerantz \& Schwaitzberg, 1975) have shown that potent effects of perceptual organization on stimulus encoding appear here as well.

\section{REFERENCES}

Attneave, F. Symmetry, information, and memory for patterns. American Journal of Psychology, 1955, 68, 209-222.

BAMBER. D. Reaction times and error rates for "same"-"different" judgments of multidimensional stimuli. Perception \& Psychophysics, 1969, 6, 169-174.

BEAR, G. Figural goodness and the predictability of figural elements. Perception \& Psychophysics, 1973, 13, 32-40.

BELl. H. H., \& HANDEL. S. The role of pattern goodness in the reproduction of backward masked patterns. Journal of Experimental Psychology: Human Perception and Performance, 1976, 2, 139-150.

Checkosky, S. F., \& Whitlock, D. Effects of pattern goodness on recognition time in a memory search task. Journal of Experimental Psychology, 1973, 100, 341-348.

Clement. D. E. Uncertainty and latency of verbal naming responses as correlates of pattern goodness. Journal of Verbal Learning and Verbal Behavior. 1964, 3, 150-157.

Clement, D. E., \& Varnadoe, K. W. Pattern uncertainty and the discrimination of visual patterns. Perception \& Psychophysics, 1967, 2, 427-431.

GaRnER, W. R. The stimulus in information processing. American Psychologist, 1970, 25, 350-358.

GARNER, W. R. The processing of information and structure. Potomac City, Md: Erlbaum. 1974.

Garner. W. R., \& Clement, D. E. Goodness of pattern and pattern uncertainty. Journal of Verbal Learning and Verbal Behavior. 1963, 2, 446-452.

Garner, W. R., \& Sutliff, D. The effect of goodness on encoding time in visual pattern discrimination. Perception \& Psychophysics, 1974, 16, 426-430.

Kornblum, S. Sequential effects in choice reaction time: A tutorial review. In S. Kornblum (Ed.) Attention and performance IV. New York: Academic Press, 1973.
Lockhead, G. R. Processing dimensional stimuli: A note. Psychological Review, 1972, 79, 410-419.

MorTon, J. Interaction of information in word recognit $n$ Psychological Review, 1969, 76, 165-178.

NeIsser, U. Cognitive psychology. New York: AppletonCentury-Crofts, 1967.

Pomerantz, J. R., \& Garner, W. R. Stimulus configuration in selective attention tasks. Perception \& Psychophysics, 1973, 14, 565-569.

Pomerantz, J. R., \& Schwaitzberg, S. D. Grouping by proximity: Selective attention measures. Perception \& Psychophysics, 1975, 18, 355-361.

STERNBERG, S. High-speed scanning in human memory. Science, 1966, 153, 652-654.

STERNBERG, S. Two operations in character recognition: Some evidence from reaction-time measurements. Perception \& Psychophysics, 1967, 2, 45-53.

STERnBERG, S. The discovery of processing stages: Extensions of Donder's method. Acta Psychologica, 1969. 30. 276-315.

\section{NOTES}

1. Let us alter the above model somewhat. Assume that probe goodness affects not pathway length but the criterion for a logogen to fire. More specifically, assume that logogens for poor patterns have higher thresholds for firing than do logogens for good ones. This model predicts that the effects of probe goodness and probe contrast are affecting the same stage of processing. Yet it is not intuitively clear why goodness and contrast affect just one stage in this model, but affect two separate stages in the previous model. This exercise points out the dangers, noted by Sternberg (1969), of applying the additive factors method in the absence of a specific a priori conceptualization of which stages are involved in performing a task and of which factors should affect which stages.

2 . Such an interaction would have been expected if goodness were a dimension of pattern similarity, that is, if patterns of differing goodness were more dissimilar and so more discrimnable from each other. Checkosky and Whitlock did find one similarity effect that was somewhat related to goodness, namely, that RTs were particularly long to nontargets that were identical to targets, except for a change in orientation (i.e., to nontargets). But, given that targets and nontargets were from different equivalence sets, there was no consistent effect in their data of whether targets and nontargets were of equal goodness.

(Received for publication April 13, 1976; revision received November $1,1976$. .) 